This paper has been published in BMJ Supportive \& Palliative Care:

Signorelli, C., Wakefield, C., McLoone, J. K., Fardell, J., Jones, J. M., Turpin, K. H., . . Cohn, R. (2019). Childhood cancer survivorship: barriers and preferences. BMJ Supportive \& Palliative Care, bmjspcare-2019-002001. doi:10.1136/bmjspcare-2019-002001; http://dx.doi.org/10.1136/bmjspcare-2019-002001

REGULAR ARTICLE

\title{
Childhood cancer survivorship: barriers and preferences
}

Christina Signorelli $(\mathrm{PhD}),{ }^{1,2}$ Claire E. Wakefield $(\mathrm{PhD}),{ }^{1,2}$ Jordana K. McLoone $(\mathrm{PhD}),{ }^{1,2}$ Joanna E. Fardell $(\mathrm{PhD}),{ }^{1,2}$ Janelle M. Jones $(\mathrm{PhD}),{ }^{3,4}$ Kate H. Turpin $(\mathrm{MNg}(\mathrm{NPrac})),{ }^{5}$ Jon Emery (DPhil),${ }^{6}$ Gisela Michel $(\mathrm{PhD}){ }^{7}$ Peter Downie (FRACP),${ }^{8}$ Jane E. Skeen (MBChB) ${ }^{9}$ and Richard J. Cohn (FRACP),${ }^{1,2}$ on behalf of the ANZCHOG Survivorship Study Group*

1. Kids Cancer Centre, Sydney Children's Hospital, NSW, Australia

2. School of Women's and Children's Health, UNSW Sydney, NSW, Australia

3. Australian and New Zealand Children's Haematology/Oncology Group, VIC, Australia

4. Hudson Institute of Medical Research, VIC, Australia

5. Paediatric Haematology/Oncology, Women's \& Children's Hospital, SA, Australia

6. Centre for Cancer Research, University of Melbourne, Victorian Comprehensive Cancer Centre, VIC, Australia

7. University of Lucerne, Department of Health Sciences and Health Policy, Switzerland

8. Children's Cancer Centre, Royal Children's Hospital, Melbourne, VIC, Australia

9. Starship Blood and Cancer Centre, Starship Children's Hospital, Auckland, New Zealand

*The members of the ANZCHOG Survivorship Study Group in alphabetical order: Dr Frank Alvaro, Prof Richard Cohn, Dr Rob Corbett, Dr Peter Downie, Ms Karen Egan, Ms Sarah Ellis, Prof Jon Emery, Dr Joanna Fardell, Ms Tali Foreman, Dr Melissa Gabriel, Prof Afaf Girgis, Ms Kerrie Graham, Ms Karen Johnston, Dr Janelle Jones, Dr Liane Lockwood, Dr Ann Maguire, Dr Maria McCarthy, Dr Jordana McLoone, Dr Francoise Mechinaud, Ms Sinead Molloy, Ms Lyndal Moore, Dr Michael Osborn, Dr Christina Signorelli, Dr Jane Skeen, Dr Heather Tapp, Ms Tracy Till, Ms Jo Truscott, Ms Kate Turpin, Prof Claire Wakefield, Ms Jane Williamson, Dr Thomas Walwyn, and Ms Kathy Yallop.

Short running title: Survivors' follow-up care barriers and preferences

Correspondence: Dr Christina Signorelli, Kids Cancer Centre, Level 1 South, Sydney Children's Hospital, High St, Randwick, NSW 2031, Sydney, AUSTRALIA; School of Women's and Children's Health, UNSW Sydney, Kensington, New South Wales, AUSTRALIA. Tel: 0061-2-9382- 5563. Fax 0061-2-9382-1789; Email: c.signorelli@unsw.edu.au

Financial disclosure: The authors have no financial relationships relevant to this article to disclose.

Funding: Christina Signorelli and Joanna Fardell are supported by The Kids' Cancer Project. Claire Wakefield is supported by a Career Development Fellowship from the NHMRC of Australia (APP1143767). The Behavioural Sciences Unit (BSU) is proudly supported by the Kids with Cancer Foundation. The BSU's survivorship research program is funded by the Kids Cancer Alliance, The Kids' Cancer Project and a Cancer Council NSW Program Grant 
(PG16-02) with the support of the Estate of the Late Harry McPaul. ANZCHOG is supported by the Australian Government, through Cancer Australia's Support for Cancer Clinical Trials program. These funding bodies did not have any role in the study, nor did they have a role in the writing of the manuscript or the decision to submit it for publication.

Conflict of interest: No author has any conflicts of interest to disclose in relation to this manuscript.

Abbreviations:

PCP - primary care physician

ANZCHOG - Australian and New Zealand Children's Haematology/Oncology Group SCP - survivorship care plan

ALL - Acute Lymphoblastic Leukemia

AYAs - adolescents and young adults

Table of Contents Summary: This multi-center study includes quantitative data of survivors' barriers to care and preferences for future care, complemented by qualitative data to further explain the findings.

\section{What is known about this topic:}

1. Many survivors are disengaged from survivorship care, mandating alternative models of follow-up care to ensure survivors receive appropriate late effects surveillance and management.

2. Barriers to accessing follow-up highlight the need for innovative solutions to promote follow-up engagement and reduce the long-term burden of cancer.

3. Comprehensive data on childhood cancer survivors' preferences for follow-up to further promote engagement, and potentially changing preferences across the survivorship trajectory, remains unknown.

\section{What this study adds:}

1. Our findings offer insight into the optimal delivery of survivorship care from survivors'/parents' perspectives.

2. Given many high-risk survivors are disengaged from cancer-related care, identifying patient preferences can be used to re-engage survivors who should be receiving cancer-related follow-up. 


\section{Contributors' Statement Page}

Christina Signorelli contributed to the study design and design of the data collection instruments, collected data, carried out the analyses, drafted the manuscript and coordinated revisions.

Claire Wakefield and Richard Cohn conceptualized and designed the study, designed the data collection instruments, coordinated and supervised data collection, reviewed and revised the manuscript.

Jordana McLoone conceptualized and designed the study, designed the data collection instruments, contributed to data collection, reviewed and revised the manuscript.

Joanna Fardell contributed to data collection, the interpretation of the data, manuscript writing and critically reviewed and revised the manuscript.

Janelle Jones, Kate Turpin, Jon Emery, Gisela Michel, Peter Downie and Jane Skeen contributed to the interpretation of the data, manuscript writing and critically reviewed and revised the manuscript.

All authors approved the final manuscript as submitted and agree to be accountable for all aspects of the work. 


\section{ABSTRACT (249 words)}

Objective: Many survivors are disengaged from follow-up, mandating alternative models of survivorship-focused care for late effects surveillance. We explored survivors' barriers to accessing, and preferences for survivorship care.

Methods: We invited Australian and New Zealand survivors of childhood cancer from three age groups: $<16$ years (represented by parents), 16-25 years (adolescent and young adults, 'AYAs'), and $>25$ years ('older survivors'). Participants completed questionnaires and optional interviews.

Results: 633 survivors/parents completed questionnaires: 187 parents of young survivors (mean age: 12.4 years), 251 AYAs (mean age: 20.6 years), and 195 older survivors (mean age: 32.5 years). Quantitative data was complemented by 151 in-depth interviews. Most participants, across all age groups, preferred specialized follow-up (i.e. involving oncologists, nurses or a multidisciplinary team; 86-97\%). Many (36-58\%) were unwilling to receive community-based follow-up. More parents (75\%) than AYAs (58\%) and older survivors $(30 \%)$ were engaged in specialized follow-up. While follow-up engagement was significantly lower in older survivors, survivors' prevalence of late effects increased. Of those attending a follow-up clinic, $34-56 \%$ were satisfied with their care, compared with $14-15 \%$ of those not receiving cancer-focused care $(p<0.001)$. Commonly reported barriers included lack of awareness about follow-up availability (67\%), followed by logistical $(65 \%)$, care-related beliefs $(59 \%)$, and financial reasons $(57 \%)$. Older survivors $(p<0.001)$, living outside major cities $(p=0.008)$, and who were further from diagnosis $(p=0.014)$ reported a higher number of barriers.

Conclusions: Understanding patient-reported barriers, and tailoring care to survivors' followup preferences, may improve engagement with care and ensure that the survivorship needs of this population are met.

\section{KEYWORDS}

Barriers; pediatric oncology; survivorship; models of care; long-term follow-up; patient preferences 
The long-term impact of childhood cancer is substantial. Survival rates have improved, however reports of late treatment-related morbidities affecting childhood cancer survivors have also proliferated. Up to $88 \%$ of survivors experience 'late effects', often decades after completing treatment for childhood cancer. ${ }^{1}$ Late effects include second neoplasms, potentially life-threatening organ dysfunction, delayed growth/development, neurocognitive impairment, and psychosocial difficulties. ${ }^{1}$ Survivors can have poor knowledge about their diagnosis and treatment, and often underestimate their risk of late effects. ${ }^{2}$ Ongoing long-term follow-up care for survivors is therefore strongly recommended to monitor for, and where possible prevent, secondary complications by educating survivors about a healthy lifestyle after treatment for cancer.

It is concerning that as few as $23 \%$ of survivors attend cancer-related follow-up care. ${ }^{3,4}$ Barriers to accessing follow-up care may relate to patient- (e.g. low perceived risk of late effects), provider- (e.g. poor physician knowledge about childhood cancer), and systemrelated factors (e.g. limited health insurance). ${ }^{5}$ Few facilities offer follow-up beyond the pediatric age group, adding to the complexity of meeting survivors' unique follow-up needs. ${ }^{6}$ Survivors usually transition from pediatric to adult care as adolescents, or young adults, at which point many become disengaged. ${ }^{7}$ With time, survivors are more likely to become disengaged from follow-up care, despite the fact that late effects increasingly emerge as they age. $^{8}$

Research suggests that survivors who attend long-term follow-up care have better longterm physical and psychosocial health outcomes than non-attendees. ${ }^{9}$ Poor follow-up engagement rates necessitate the development and implementation of improved models of survivorship care. Various models are recommended in the literature, including oncologist-, nurse-, or primary care physician-led (PCP) or a shared-care model. ${ }^{10}$ The success of any model of survivorship care critically depends on establishing stakeholders' preferences. ${ }^{11}$ 
Few data are available about the preferences of childhood cancer survivors and parents, a crucial gap in informing the development of new models of survivorship care.

Our study had two primary aims. The first was to characterize the nature and prevalence of patient-reported barriers to accessing follow-up care, and assess any clinical/demographic factors associated with survivor-reported barriers. The second was to explore survivors' and parents' preferences for the delivery of survivorship care, including their preferred health professional to lead their care, mode of delivery, location, timing, and reminder methods and which professionals they would like to access for follow-up in a multidisciplinary team. We focused on these aspects because they are modifiable in considering future models of care, and research suggests that organizational aspects (e.g. appointment times) are a key priority for survivors, significantly influencing their satisfaction with care. ${ }^{12}$

To gain a more balanced and comprehensive understanding of consumers' preferences for care, our study uniquely involved parents of young survivors, and young and older survivors, in both early and late survivorship. We purposely recruited survivors who were regular attendees at follow-up, and survivors who were not engaged in any cancer-related follow-up.

\section{METHODS}

We obtained ethics approval from local boards (South Eastern Sydney Local Health District, Ref:12/POWH/345) and written consent from all participants. We implemented a sequential explanatory mixed-methods study design, collecting data in two stages. We invited parents/survivors from 11 hospitals in Australia/New Zealand to complete a questionnaire as part of the Australian and New Zealand Children's Haematology/Oncology Group (ANZCHOG) Survivorship Study. ${ }^{6}$ Questionnaire respondents could opt-in for an optional interview. 


\subsection{Participants}

In order to capture potentially differing preferences across age groups, we recruited: a) parents of young survivors $<16$ years ['parents'], b) adolescent and young adult (AYA) survivors of childhood cancer aged 16-25 years ['AYA survivors'], and c) survivors of childhood cancer aged over 25 years old ['older survivors']. Survivors were eligible if they were diagnosed with cancer before the age of 16 , were at least 5 years from diagnosis, were in remission, and were treated at an Australian/New Zealand hospital.

\subsection{Data collection}

We searched for eligible survivors in participating hospitals' electronic medical records. We posted invitations, questionnaires, and reply-paid envelopes and conducted telephone follow-up four weeks after mail-out. For survivors $<16$ years, invitations were addressed to parents. We posted a second information package to questionnaire respondents who opted in for interviews, containing a consent form and reply-paid envelope. We telephoned interested survivors/parents to arrange an interview time. Experienced researchers who had no prior relationship with the participants (CS, JKM, JEF) conducted the one-on-one interviews. We did not offer interviewees the opportunity to review their transcripts.

\subsection{Measures (see Supplementary File 1 for detail)}

We collected participants' demographic and clinical information (Table 1), as well as the number of late effects survivors reported experiencing since treatment (Supplementary File 2). We measured barriers to follow-up engagement and participants' satisfaction with care. Participants indicated their preferred health professional (e.g. oncologist, PCP), location (e.g. non-hospital facility), delivery mode (e.g. phone, online), appointment reminders (e.g. text), timing and access to services.

\subsection{Statistical analysis}


We analyzed quantitative data using SPSS24.0, considering results statistically significant when $\mathrm{p}<0.05$ (two-tailed). To describe sample characteristics and compare barriers and preferences for follow-up across groups we used descriptive statistics, chi-squared tests, and analysis of variance. We used multivariate linear regressions to analyze demographic and clinical factors associated with the total number of barriers to accessing follow-up care. We included variables in the multivariate regression that were statistically significant at $p<0.2$ in univariate models.

We audio-recorded and transcribed interviews verbatim. We conducted directed qualitative content analysis using NVivo11. Two researchers (CS, MEB) read transcripts alongside data collection and discussed emergent categories to develop a coding tree, based on predetermined themes, informed by the study aims and guided by the Miles and Huberman methodology. ${ }^{13}$ Two coders (CS, MEB) coded $20 \%$ of randomly selected interviews. Discrepancies were resolved through discussion and amendments made to the coding tree as needed. Given that concordance was high (96.8\%) and the scale of the study, one coder (CS) analyzed the remainder of the data. We used qualitative data to further explain the quantitative findings, stopping data collection once we achieved data saturation.

\section{RESULTS}

Of 900 eligible and contactable parents/survivors who we invited, 633 completed questionnaires (71\% response rate): 187 parents of young survivors $(<16$ years old; survivors' mean age:12.4 at study participation; 9.7 years on average from diagnosis), 251 AYAs (16-25 years; mean age:20.6; 14.3 years from diagnosis), and 195 older survivors ( $>25$ years old; mean age:32.5; 24.6 years from diagnosis). Table 1 details participant characteristics.

Questionnaire respondents were more likely to be female $(51.7 \%$ vs $43.9 \%, p=0.009)$; and older (mean age:21.5 vs $20.1, \mathrm{t}(1150)=-2.827, p=0.005)$ than non-respondents. Of the 
questionnaire respondents, 151 also completed optional telephone interviews. There was no significant difference between interviewee respondents and non-respondents in sex $(p=0.645)$, however interview respondents were younger than non-respondents (mean age: 19.7 vs $22.0, \mathrm{t}(624)=2.870, p=0.004)$.

More parents (75\%) and AYAs (58\%) than older survivors (30\%) were engaged in follow-up care $(p<0.001)$. Sixty-five percent of parents, $68 \%$ of AYAs, and $64 \%$ of older survivors reported having a regular PCP. Figure 2 demonstrates that older survivors' followup attendance was lower, whilst the mean prevalence of late effects was higher. Participants in all age groups who were not currently receiving specialized follow-up (i.e. hospital-based, oncologist-led, or multidisciplinary team follow-up), reported significantly greater dissatisfaction with their care (34-56\%) than those who were engaged $(14-15 \%, p<0.001$; Figure 3). Supplementary File 3 contains illustrative quotations further explaining key barriers and preferences for care.

\subsection{Barriers to care}

The most commonly endorsed barriers related to lack of awareness of follow-up availability (67\%), followed by logistical (65\%), belief-related (59\%), and financial $(57 \%)$ reasons. On average, parents endorsed fewer barriers to accessing care (mean:4.2) than AYAs (mean:6.3) or older survivors (mean:6.5; $\mathrm{F}(2,625)=16.103, p<0.001$ ). We found no difference in the average number of barriers endorsed by parents $(\mathrm{p}=0.410)$ or AYAs $(\mathrm{p}=0.333)$ living in rural/remote areas compared with metropolitan areas. However, older survivors in rural/remote areas endorsed more barriers than survivors in metropolitan areas (mean:5.2 versus $4.6 ; \mathrm{t}(172)=-2.781, p=0.006)$.

Survivors who were disengaged from cancer-related care endorsed all barriers more frequently than those who attended follow-up (all $\mathrm{p}<0.05$ ), with the exception of an 'inability to travel without assistance' and 'aversion to tests' (Figure 1). Survivors who reported being 
dissatisfied with their follow-up endorsed significantly more barriers in total (mean $=6.5$ ) than those who were satisfied with their care $($ mean $=5.3 ; \mathrm{t}(608)=3.152, p=0.002)$.

Supplementary File 4 details the univariate and multivariate regression results, assessing demographic and clinical factors associated with participants' total number of follow-up barriers. In multivariate regression analysis, survivors tended to report a greater number of barriers if they were older $(\beta=2.10,95 \% \mathrm{CI}=1.24-2.95, p<0.001)$, lived outside a major city $(\beta=1.22,95 \% \mathrm{CI}=0.32-2.12, p=0.008)$, and were further from their primary diagnosis $(\beta=0.06,95 \% \mathrm{CI}=0.01-0.11, p=0.014)$. No other demographic (e.g. sex, ethnicity) or clinical factors (e.g. diagnosis, treatment) were associated with the number of barriers reported.

\subsection{Survivorship care preferences}

\subsubsection{Preferred follow-up professionals}

Hospital-based survivorship care - involving an oncologist or other clinic doctor, cancer survivorship nurse or team - was most commonly endorsed as the 'first choice' across all age groups (97\% parents, 88\% AYAs, 86\% older survivors; Figure 4). Primary-based follow-up was least preferred, with many stating that they were unwilling to visit a PCP for cancerrelated follow-up (parents: 58\%, AYAs: 36\%, older survivors: 35\%). A reliance on oncologists, and reluctance to visit PCPs, was supported by interview data suggesting that participants had lower confidence in PCPs who were less involved during cancer treatment: "I would probably be more confident in seeing [my oncologist], because she was there...from when I was diagnosed to when I finished" (female Neuroblastoma survivor, 25 years old).

Besides oncologists, the healthcare professional most desired to be available in a follow-up clinic differed by age (Supplementary File 5). Among parents and AYAs, the two most requested professionals were psychologists (64\% and 55\% respectively) and fertility specialists (60\% and 54\%). Parents' third request was for access to dentists $(58 \%)$, while 
AYAs' third-most requested professional was PCPs (53\%). Among older survivors, fertility specialists were most requested (62\%), followed by PCPs (56\%) and psychologists (54\%).

\subsubsection{Preferred follow-up mode}

The preferred method of delivery for follow-up consultations was face-to-face $(81 \%$ parents, $75 \%$ AYAs, $64 \%$ older survivors), followed by telephone follow-up (13\%, 18\% and 25\% respectively) or questionnaire assessment with a face-to-face appointment only if needed $(6 \%, 7 \%$ and $11 \%$ respectively). As one parent summarized "sometimes face-to-face is better. But at the same time I certainly think that when it's appropriate using [online] tools is a really good idea from an efficiency point of view" (father of Hepatoblastoma survivor, aged 14).

\subsubsection{Preferred follow-up location}

Eighty-eight percent of parents preferred follow-up to be delivered in a pediatric outpatient hospital setting, but fewer AYAs (54\%) and older survivors (29\%) identified this as their 'first choice'. An adult outpatient hospital setting was the most commonly endorsed location for older survivors (42\%), who reported being uncomfortable in a pediatric setting: "I am now 30 years old, so attending clinics with this age group is uncomfortable. I would like to meet or attend with people my age" (female Acute Lymphoblastic Leukemia [ALL] survivor, aged 30).The least endorsed option among all groups was a stand-alone clinic at a university or other non-hospital facility (3-15\%).

Most participants reported feeling comfortable receiving follow-up care in a clinic with patients still receiving active treatment (68\% parents, $74 \%$ AYAs, $66 \%$ older survivors). For the remainder of participants, clinics run in an acute treatment setting was a disincentive to attend. Qualitatively, participants receiving care in any hospital setting meant facing "the memories that my childhood treatment bring[s] back" (male ALL survivor aged 44).

\subsubsection{Preferred follow-up timing}


Most parents (66\%) were willing to attend follow-up with their child during working hours. However, $49 \%$ of AYAs and older survivors requested follow-up clinic be held afterhours. Some families suggested "making times more flexible” (Mother of Neuroblastoma survivor, aged 9) would improve attendance to allow travel at more convenient times, for example avoiding school drop-off/pick-up times.

\subsubsection{Preferred approach to reminders}

Participants in all groups preferred reminders for follow-up appointments directly from the clinic, specifically letters (51-60\% of participants), emails (50-60\%) or text messages $(34-50 \%)$. Few indicated that their partners (2-3\%) or their parents/child (4-16\%) were their preferred source of reminders. When left to remember clinic appointments themselves this made some participants feel "we are less important and a bit of a nuisance" (Mother of ALL survivor, aged 8). Participants also suggested calendar reminders through an online application/program that contained the patients' treatment plan/summary.

\section{DISCUSSION}

This is the first Australian/New Zealand study evaluating survivors'/parents' barriers to attending, and preferences for, survivorship care. Attendance in our young survivors was $75 \%$, with lower attendance in AYAs (58\%) and older survivors (30\%). We found that older survivors, living outside major cities, and survivors who were a longer time from diagnosis, were more likely to endorse a higher average number of barriers to engaging in follow-up. Our findings suggest that the survivorship care preferences of parents, AYAs, and older survivors are mostly similar. Most participants indicated a preference for face-to-face hospital-based care. The most requested additional health professionals at survivorship clinics were psychologists and fertility specialists.

Follow-up engagement in our study was sub-optimal (30-75\%), mirrored in long-term survivors globally. ${ }^{14-16}$ Commonly endorsed barriers related primarily to low follow-up 
awareness, low perceived risk, and logistical factors, reinforcing research suggesting that non-financial barriers significantly hinder follow-up adherence. ${ }^{17}$ Low follow-up clinic awareness may indicate a group of survivors who have missed the educational opportunities on survivorship including navigating the healthcare system. Around two-thirds of survivors endorsed logistical barriers in our study, many of which are modifiable through simple intervention (e.g. appointment reminders). Survivors with higher perceived barriers are less likely to attend follow-up, highlighting the need to address practical barriers. ${ }^{18}$ Survivors in our cohort who lived outside major cities reported a greater number of barriers. Research shows that a greater distance travelled to follow-up is associated with diminished attendance rates, ${ }^{14}$ echoed in our qualitative findings. Distance-related barriers may be perpetuated by Australia/New Zealand's highly dispersed population, centralization of clinics, and clinic's broad catchment areas. ${ }^{19}$

Participants preferred hospital-based to community-based follow-up as their first choice, possibly reflecting early detachment from PCPs during treatment and lack of exposure to alternative models. ${ }^{20,21}$ However, a purely hospital-based model is not sustainable as the number of survivors increases. ${ }^{6,22}$ Risk-stratification may address the pitfalls of this model, and limited global resources, by triaging high-risk/complex survivors to multidisciplinary specialist-led care and lower risk survivors to shared or nurse/PCP-led care. ${ }^{23,24}$ However, the implementation of risk-based models requires that survivors and health professionals are both willing and confident participants. Whilst PCPs seem willing to deliver childhood survivorship care, up to $58 \%$ of survivors in our study were unwilling to receive community-based follow-up, potentially reflecting survivors' emotional connection to their oncology team. ${ }^{21,25,26}$ Additional PCP education, support, communication, ${ }^{27}$ and a "culture change" to establish expectations from diagnosis may be required to ensure the success of community-based models. ${ }^{26}$ 
Our data echoes existing literature showing that many survivors who are at high risk of developing late effects (e.g. second malignancies, cardiovascular disease) are disengaged from cancer-related care. ${ }^{22,23}$ Our study highlights the alarming pattern of declining engagement in follow-up care over time, despite survivors' escalating risk of developing late effects, resulting in a significant gap in care. Additional research is therefore needed to evaluate interventions that promote follow-up adherence, ${ }^{28}$ particularly in age cohorts where this disparity is greatest. To achieve this, it is critical to address patient-reported barriers and preferences to ensure older survivors remain engaged in care, and to mitigate poor patient outcomes and the associated healthcare system costs. A significant minority of survivors in our study (up to $25 \%$ ) were open to non-traditional follow-up (e.g. telephone/questionnaire). Innovative solutions, including postal health questionnaires, ${ }^{29,30}$ internet-based, ${ }^{31,32}$ and telephone/video-conferencing interventions, ${ }^{33}$ may help to promote follow-up engagement by overcoming reported barriers.

Future models should address the complex and varying needs of this population, indicated by the diversity of health professionals to whom our participants requested access. High demand for psychologists highlights the long-term challenges necessitating psychosocial support, emphasized in the release of recent guidelines. ${ }^{34}$ Traditional risk-based models may not fully account for psychological late effects for example referring medically 'low-risk' survivors to primary care where psycho-oncology care is not routine. Psychological support may also help to overcome barriers transitioning from pediatric to adult follow-up care. ${ }^{35}$

Differing preferences across age groups may be explained by highly varying follow-up practices across Australia/New Zealand, for example discharge age from paediatric follow-up care. ${ }^{6}$ Variations in the type and quality of care survivors have previously received, ${ }^{36}$ or their prior/current engagement patterns, ${ }^{37}$ could shape their preferences and 
openness to alternative models. This adds to the complexity of conceptualizing future models of care that address key barriers and capture patient preferences.

This multi-center study includes quantitative and qualitative data of survivors' preferences. Our sample captured follow-up attendees and non-attendees, many of whom were several decades from treatment. As is common in this field, ${ }^{38}$ most participating parents were mothers, leaving fathers' opinions underrepresented. Our questionnaire omitted options such as AYA specialized clinics, reflecting local practices at the time of the study. Results nonetheless indicated that many AYAs were dissatisfied with their follow-up, echoing the need for age-appropriate models. ${ }^{39}$ Our study included a wide age range of survivors. Grouping older survivors as $>25$ years may have masked unique preferences in much older survivors (e.g., over 40s). Longitudinal studies are needed to better capture the nature of changes in barriers and preferences over time.

\section{CONCLUSION}

This study evaluated patient-reported barriers to accessing follow-up care, and survivors' and parents' preferences for the delivery of survivorship care. It is critical to establish practices which align with patient preferences to encourage engagement in care and better ensure patients' survivorship needs are met. Partnering with survivors and families will ensure we meet the survivors' complex needs through coordinated and well-designed care. Innovative solutions may be required to overcome patient-reported barriers and align with families' preferences, whilst meeting the needs of resource-limited healthcare environments.

Acknowledgements: We would like to thank Dr Kate Hetherington, Dr Janine Vetsch, and Brittany McGill who assisted with interviews, and Mary-Ellen Brierley who conducted interviews and assisted with qualitative data analysis. We would also like to thank the families who participated and each of the recruiting sites including: Sydney Children's Hospital Randwick, the Children's Hospital at Westmead, John Hunter Children's Hospital, the Royal Children's Hospital Melbourne, Monash Children's Hospital Melbourne, Lady Cilento Children's Hospital Brisbane (formerly the Royal Children's Hospital Brisbane), Perth Children's Hospital (formerly Princess Margaret Children's Hospital Perth), Women's 
and Children's Hospital Adelaide, and in New Zealand, Starship Children's Hospital, Wellington Hospital and Christchurch Hospital. We acknowledge the Department of Health Western Australia and Data Linkage Branch for access to participants' vital status information. 


\section{References}

1. Phillips SM, Padgett LS, Leisenring WM, et al. Survivors of childhood cancer in the United States: prevalence and burden of morbidity. Cancer Epidemiology and Prevention Biomarkers. 2015;24(4):653-663.

2. Carretier J, Boyle H, Duval S, et al. A review of health behaviors in childhood and adolescent cancer survivors: toward prevention of second primary cancer. Journal of adolescent and young adult oncology. 2016;5(2):78-90.

3. Miller KA, Wojcik KY, Ramirez CN, et al. Supporting long-term follow-up of young adult survivors of childhood cancer: Correlates of healthcare self-efficacy. Pediatric blood \& cancer. 2017;64(2):358-363.

4. Wilson CL, Cohn RJ, Johnson KA, Ashton LJ. Tracing survivors of childhood cancer in Australia. Pediatric blood \& cancer. 2009;52(4):510-515.

5. Essig S, Skinner R, Nicolas X, Kuehni CE, Michel G. Follow-up programs for childhood cancer survivors in Europe: a questionnaire survey. PLoS One. 2012;7(12):e53201.

6. Signorelli C, Wakefield CE, McLoone JK, et al. Models of childhood cancer survivorship care in Australia and New Zealand: Strengths and challenges. Asia-Pacific Journal of Clinical Oncology. 2017.

7. Henderson TO, Friedman DL, Meadows AT. Childhood cancer survivors: transition to adultfocused risk-based care. Pediatrics. 2010;126(1):129-136.

8. Rokitka DA, Curtin C, Heffler JE, Zevon MA, Attwood K, Mahoney MC. Patterns of loss to follow-up care among childhood cancer survivors. Journal of adolescent and young adult oncology. 2017;6(1):67-73.

9. Signorelli C, Wakefield CE, Fardell JE, et al. The impact of long-term follow-up care for childhood cancer survivors: A systematic review. Critical Reviews in Oncology/Hematology. 2017;114:131-138.

10. Oeffinger KC, McCabe MS. Models for delivering survivorship care. Journal of Clinical Oncology. 2006;24(32):5117-5124. 
11. Lawrence RA, McLoone JK, Wakefield CE, Cohn RJ. Primary care physicians' perspectives of their role in cancer care: a systematic review. Journal of general internal medicine. 2016;31(10):1222-1236.

12. Absolom K, Greenfield D, Ross R, et al. Predictors of clinic satisfaction among adult survivors of childhood cancer. European Journal of Cancer. 2006;42(10):1421-1427.

13. Miles MB, Huberman AM. Qualitative data analysis: An expanded sourcebook. sage; 1994.

14. Barakat LP, Schwartz LA, Szabo MM, Hussey HM, Bunin GR. Factors that contribute to post-treatment follow-up care for survivors of childhood cancer. Journal of Cancer Survivorship. 2012;6(2):155-162.

15. Casillas J, Oeffinger KC, Hudson MM, et al. Identifying predictors of longitudinal decline in the level of medical care received by adult survivors of childhood cancer: a report from the childhood cancer survivor study. Health services research. 2015;50(4):1021-1042.

16. Nathan PC, Agha M, Pole JD, et al. Predictors of attendance at specialized survivor clinics in a population-based cohort of adult survivors of childhood cancer. Journal of Cancer Survivorship. 2016;10(4):611-618.

17. Smits-Seemann RR, Kaul S, Zamora ER, Wu YP, Kirchhoff AC. Barriers to follow-up care among survivors of adolescent and young adult cancer. Journal of Cancer Survivorship. 2017;11(1):126-132.

18. Lupatsch JE, Wengenroth L, Rueegg CS, et al. Follow-up care of adolescent survivors of childhood cancer: The role of health beliefs. Pediatric blood \& cancer. 2016;63(2):318-325.

19. Daniel G, Wakefield C, Ryan B, Fleming C, Levett N, Cohn R. Accommodation in pediatric oncology: parental experiences, preferences and unmet needs. Rural \& Remote Health. $2013 ; 13(2)$.

20. Michel G, Gianinazzi M, Eiser C, et al. Preferences for long-term follow-up care in childhood cancer survivors. European journal of cancer care. 2016;25(6):1024-1033.

21. Signorelli C, Wakefield CE, Fardell JE, et al. The Role Of Primary Care Physicians in Childhood Cancer Survivorship Care: Multiperspective Interviews. The oncologist. 2018:theoncologist. 2018-0103. 
22. Warren JL, Mariotto AB, Meekins A, Topor M, Brown ML. Current and future utilization of services from medical oncologists. Journal of Clinical Oncology. 2008;26(19):3242-3247.

23. Wallace WHB, Blacklay A, Eiser C, et al. Regular review: Developing strategies for long term follow up of survivors of childhood cancer. BMJ: British Medical Journal. 2001;323(7307):271.

24. Frobisher C, Glaser A, Levitt GA, et al. Risk stratification of childhood cancer survivors necessary for evidence-based clinical long-term follow-up. British journal of cancer. 2017;117(11):1723.

25. Gray WN, Schaefer MR, Resmini-Rawlinson A, Wagoner ST. Barriers to Transition From Pediatric to Adult Care: A Systematic Review. Journal of Pediatric Psychology. 2017.

26. Mayer DK, Alfano CM. Personalized Risk-Stratified Cancer Follow-Up Care: Its Potential for Healthier Survivors, Happier Clinicians, and Lower Costs. JNCI: Journal of the National Cancer Institute. 2019.

27. Blaauwbroek R, Tuinier W, Meyboom-de Jong B, Kamps WA, Postma A. Shared care by paediatric oncologists and family doctors for long-term follow-up of adult childhood cancer survivors: a pilot study. The lancet oncology. 2008;9(3):232-238.

28. May L, Schwartz DD, Frugé E, et al. Predictors of Suboptimal Follow-up in Pediatric Cancer Survivors. Journal of pediatric hematology/oncology. 2017;39(3):e143-e149.

29. Nathan PC, Ness KK, Mahoney MC, et al. Screening and surveillance for second malignant neoplasms in adult survivors of childhood cancer: a report from the childhood cancer survivor study. Annals of internal medicine. 2010;153(7):442-451.

30. Zheng DJ, Sint K, Mitchell H-R, Kadan-Lottick NS. Patterns and predictors of survivorship clinic attendance in a population-based sample of pediatric and young adult childhood cancer survivors. Journal of Cancer Survivorship. 2016;10(3):505-513.

31. Mertens A, Williamson R, Gilleland-Marchak J, Meacham LR, Cherven B, Edwards P. Evaluation of the adoption, acceptance, and satisfaction of SurvivorLink as a communication tool for pediatric cancer survivors. American Society of Clinical Oncology; 2016. 
32. Horowitz ME, Fordis M, Krause S, McKellar J, Poplack DG. Passport for care: implementing the survivorship care plan. Journal of oncology practice. 2009;5(3):110-112.

33. Signorelli C WC, Johnston KA, Fardell J, Brierley ME, Thornton-Benko E, et al. 'Re-engage' pilot study protocol: a nurse-led eHealth intervention to re-engage, educate, and empower childhood cancer survivors. . BMJ Open. 2018 (in press).

34. Wiener L, Kazak AE, Noll RB, Patenaude AF, Kupst MJ. Standards for the psychosocial care of children with cancer and their families: an introduction to the special issue. Pediatric blood \& cancer. 2015;62(S5):S419-S424.

35. Gray WN, Monaghan MC, Marchak JG, Driscoll KA, Hilliard ME. Psychologists and the transition from pediatrics to adult health care. Journal of Adolescent Health. 2015;57(5):468474.

36. Lisy K, Emery JD, Jefford M. Provider roles in survivorship care: Moving beyond surveys of patient preference. Journal of Clinical Oncology. 2017;35(34):3890-3890.

37. Vetsch J, Rueegg C, Mader L, et al. Parents' preferences for the organisation of long-term follow-up of childhood cancer survivors. European journal of cancer care. 2017.

38. Wakefield CE, Fardell JE, Doolan EL, et al. Participation in psychosocial oncology and quality-of-life research: a systematic review. The Lancet Oncology. 2017;18(3):e153-e165.

39. Fardell J, Patterson P., Wakefield, CE., Signorelli, C., Cohn, RJ. Anazodo, A., Zebrack, B., Sansom-Daly and U.M. . A narrative review of models of care for adolescents and young adults with cancer: barriers and recommendations. . Journal of Adolescent and Young Adult Oncology. 2017 (in press). 


\section{FIGURE LEGENDS}

Table 1. Participant clinical and demographic characteristics

Figure 1. Percentage of participants who endorsed each barrier to accessing follow-up care Figure 2. Proportion of survivors engaged in cancer-related follow-up (2A) and survivors' mean number of reported late effects (2B) by age group

Figure 3. Proportion of participants engaged in hospital-based follow-up and dissatisfied with care

Figure 4. Participants' 'first choice' and willingness to see physicians for follow-up

\section{SUPPLEMENTARY MATERIAL}

Supplementary File 1. Supplemental detail of study methods

Supplementary File 2. List of late effects included in survey

Supplementary File 3. Illustrative quotations of key patient-reported barriers to care and preferences for survivorship care

Supplementary File 4. Key clinical and demographic predictors of patient-reported barriers to accessing follow-up care

Supplementary File 5. Participants' preferences for health professionals available via long term follow-up 
Table 1. Participant clinical and demographic characteristics

\begin{tabular}{|c|c|c|c|}
\hline Characteristic & $\begin{array}{c}\text { Parents of survivors }^{\mathrm{a}} \\
<16 \text { years } \\
(\mathrm{n}=187)\end{array}$ & $\begin{array}{c}\text { AYA } \\
\text { survivors } \\
\text { 16-25 years } \\
(\mathbf{n}=\mathbf{2 5 1}) \\
\end{array}$ & $\begin{array}{c}\text { Older } \\
\text { survivors } \\
>25 \text { years } \\
(\mathrm{n}=195)\end{array}$ \\
\hline Male survivors & $101(54 \%)$ & $119(48 \%)$ & $85(44 \%)$ \\
\hline Fathers of survivors & $25(14 \%)$ & NA & NA \\
\hline \multicolumn{4}{|l|}{ Self-identified ethnicity } \\
\hline Australian/New Zealand & $133(74 \%)$ & $197(81 \%)$ & $143(77 \%)$ \\
\hline Not Australian/New Zealand & $48(26 \%)$ & $45(18 \%)$ & $42(23 \%)$ \\
\hline \multicolumn{4}{|l|}{ Area of residence ${ }^{b}$} \\
\hline Major city & $127(76 \%)$ & $166(74 \%)$ & $144(81 \%)$ \\
\hline Regional/remote & $40(24 \%)$ & $57(26 \%)$ & $33(19 \%)$ \\
\hline \multicolumn{4}{|l|}{ Education } \\
\hline High school only & $49(26 \%)$ & $147(60 \%)$ & $51(27 \%)$ \\
\hline Post-school (e.g. university) & $137(74 \%)$ & $97(40 \%)$ & $139(73 \%)$ \\
\hline Employed & $134(72 \%)$ & $152(62 \%)$ & $137(73 \%)$ \\
\hline \multicolumn{4}{|l|}{ Income } \\
\hline$<$ AUD $\$ 60,000$ & $41(24 \%)$ & $145(60 \%)$ & $74(46 \%)$ \\
\hline$>$ AUD $\$ 60,000$ & $128(76 \%)$ & $62(49 \%)$ & $85(54 \%)$ \\
\hline \multicolumn{4}{|l|}{ Diagnosis } \\
\hline Leukemia & $86(46 \%)$ & $105(46 \%)$ & $79(41 \%)$ \\
\hline Lymphoma & $13(7 \%)$ & $24(10 \%)$ & $38(20 \%)$ \\
\hline Brain cancer & $9(5 \%)$ & $30(13 \%)$ & $24(13 \%)$ \\
\hline Other & $59(31 \%)$ & $71(31 \%)$ & $50(26 \%)$ \\
\hline \multicolumn{4}{|l|}{ Treatment(s) received } \\
\hline Surgery & $77(44 \%)$ & $118(50 \%)$ & $78(46 \%)$ \\
\hline Chemotherapy & $176(95 \%)$ & $231(94 \%)$ & $168(89 \%)$ \\
\hline Radiation & $47(28 \%)$ & $91(40 \%)$ & $110(61 \%)$ \\
\hline Bone Marrow transplant & $34(20 \%)$ & $46(20 \%)$ & $30(18 \%)$ \\
\hline Engaged in hospital-based follow-up & $146(78 \%)$ & $143(58 \%)$ & $58(30 \%)$ \\
\hline Regular primary care physician & $122(65 \%)$ & $169(68 \%)$ & $121(64 \%)$ \\
\hline \multirow[t]{2}{*}{ Satisfied with care } & $107(58 \%)$ & $190(78 \%)$ & $107(58 \%)$ \\
\hline & Mean (SD) & Mean (SD) & Mean (SD) \\
\hline Survivor age & $12.4(2.2)$ & $20.6(2.6)$ & $32.5(6.2)$ \\
\hline Range & $7-15$ & $16-23$ & $26-61$ \\
\hline Years' since diagnosis & $9.7(2.1)$ & $14.3(4.4)$ & $24.6(8.0)$ \\
\hline Range & $5-15$ & $5-24$ & $5-59$ \\
\hline Years' since treatment completion & $8.1(2.6)$ & $12.3(4.4)$ & $22.3(7.8)$ \\
\hline Range & $0-15$ & $0-23$ & $5-59$ \\
\hline Total number of late effects & $3.2(3.1)$ & $3.5(3.4)$ & $4.1(3.8)$ \\
\hline Range & $0-15$ & $0-17$ & $0-19$ \\
\hline $\begin{array}{l}\text { Total number of barriers to follow- } \\
\text { up }\end{array}$ & $4.2(4.0)$ & $6.3(4.4)$ & $6.5(4.7)$ \\
\hline Range & $0-19$ & $0-18$ & 0-19 \\
\hline
\end{tabular}

Abbreviations: N: Number of participants; NA: Not assessed, or not applicable; SD: Standard deviation a Parent demographic data is reported; while child survivors' clinical data is reported (parent proxy).

${ }^{b}$ According to Area of Remoteness Index Australia (ARIA) classifications. We manually categorized New Zealand postcodes according to the Statistics New Zealand Urban/Rural Profile Classifications.

Numbers and percentages may not add up due to missing values and rounding errors. 
Figure 1. Percentage of participants who endorsed each barrier to accessing follow-up care

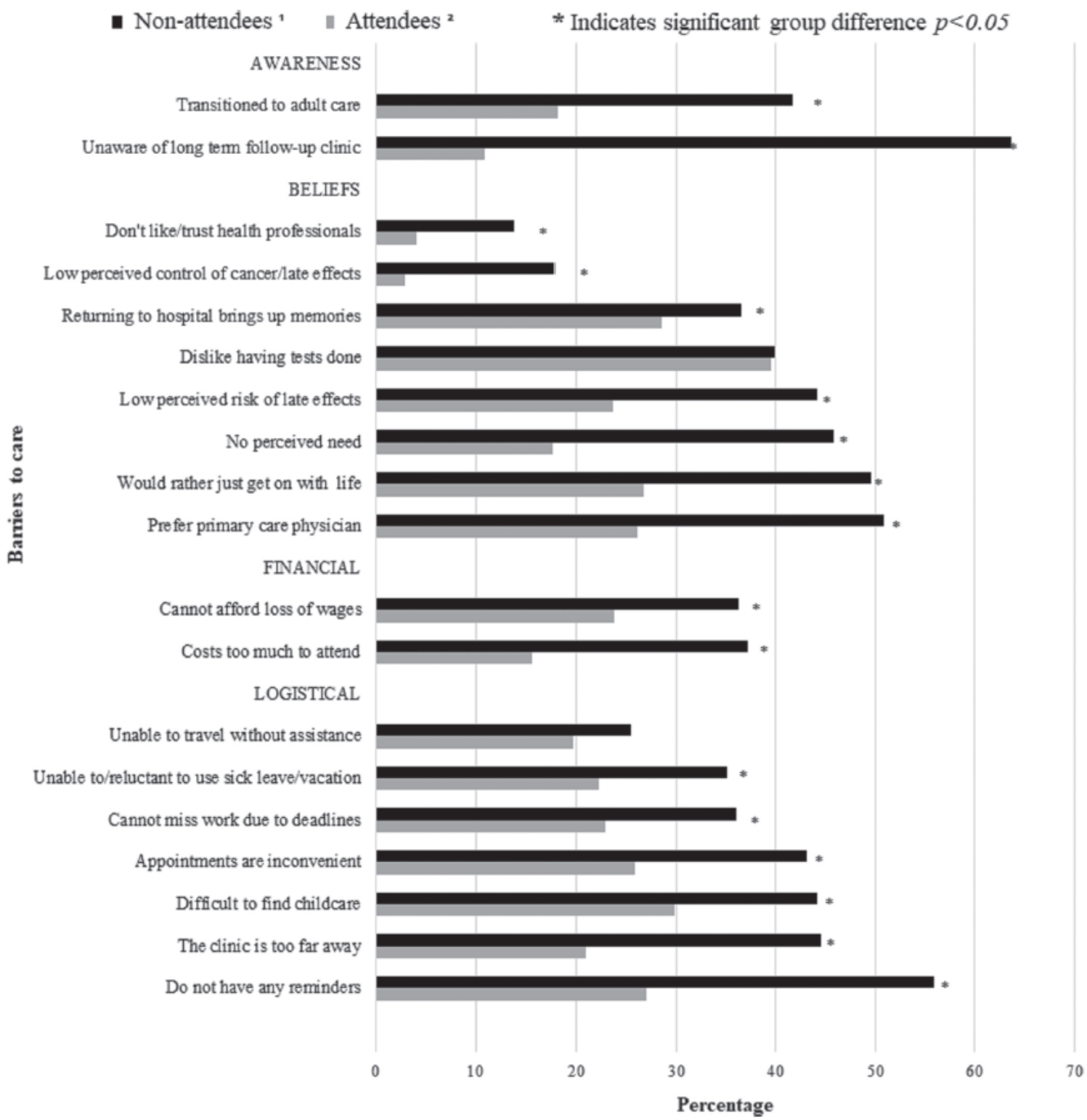


Figure 2. Proportion of survivors engaged in cancer-related follow-up (2A) and survivors' mean number of reported late effects (2B) by age group

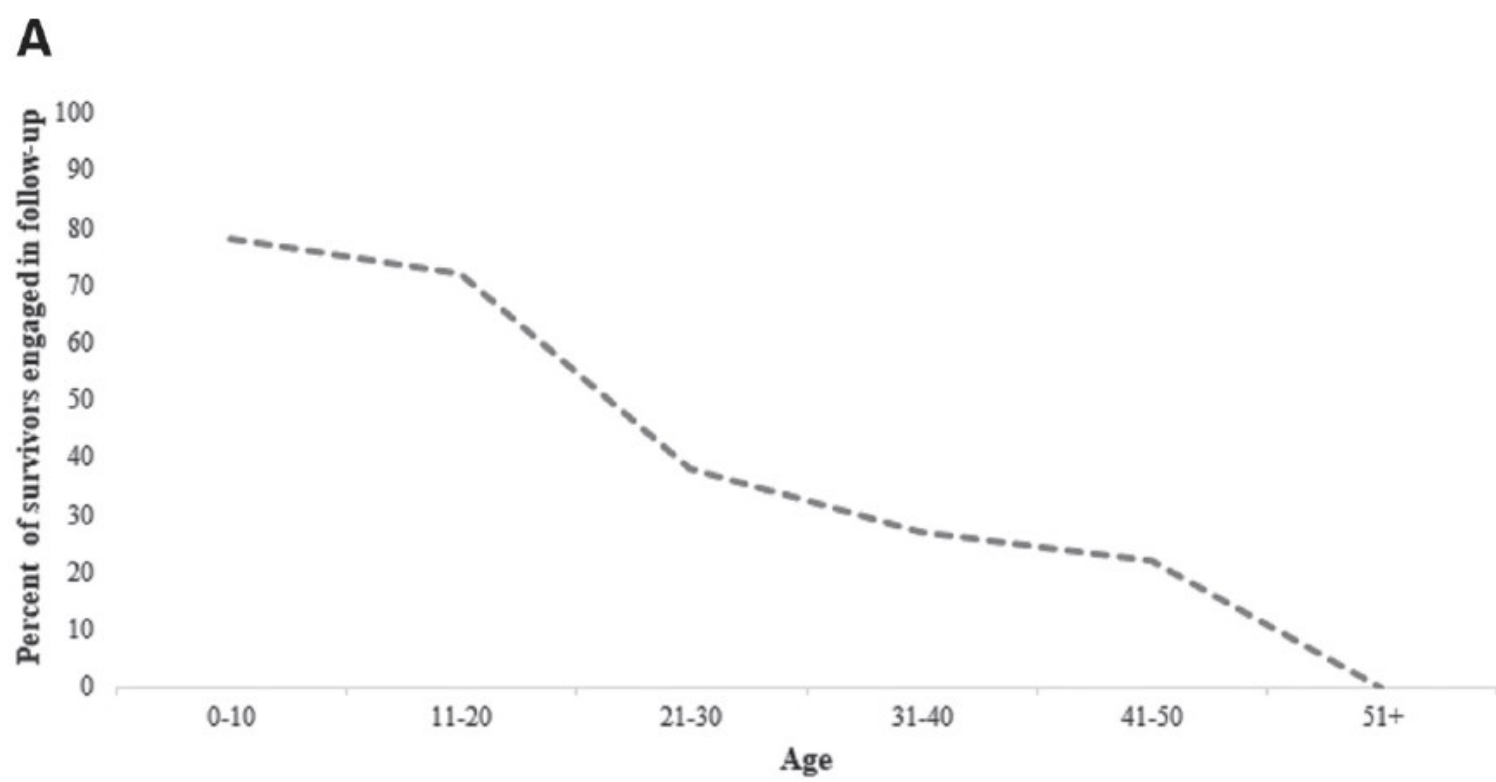

B

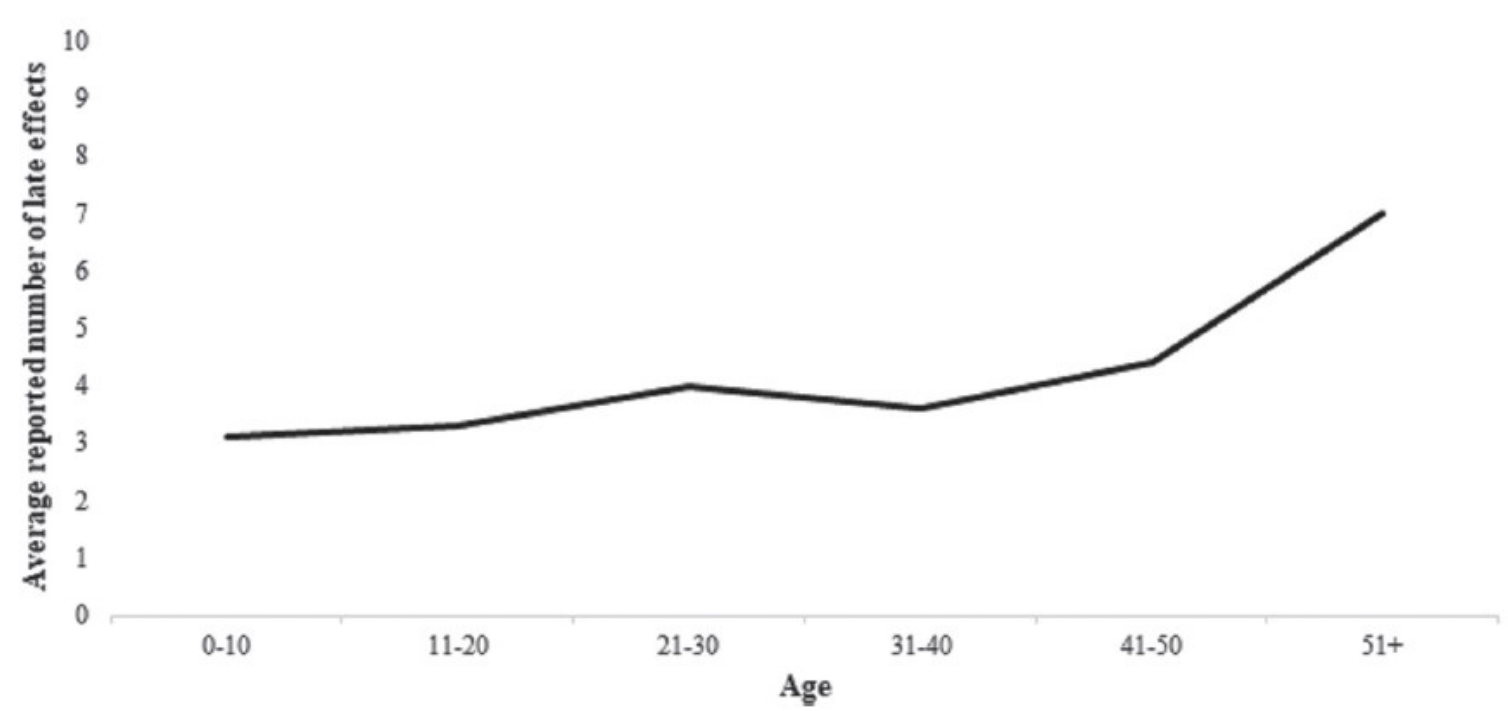


Figure 3. Proportion of participants engaged in hospital-based follow-up and dissatisfied with care

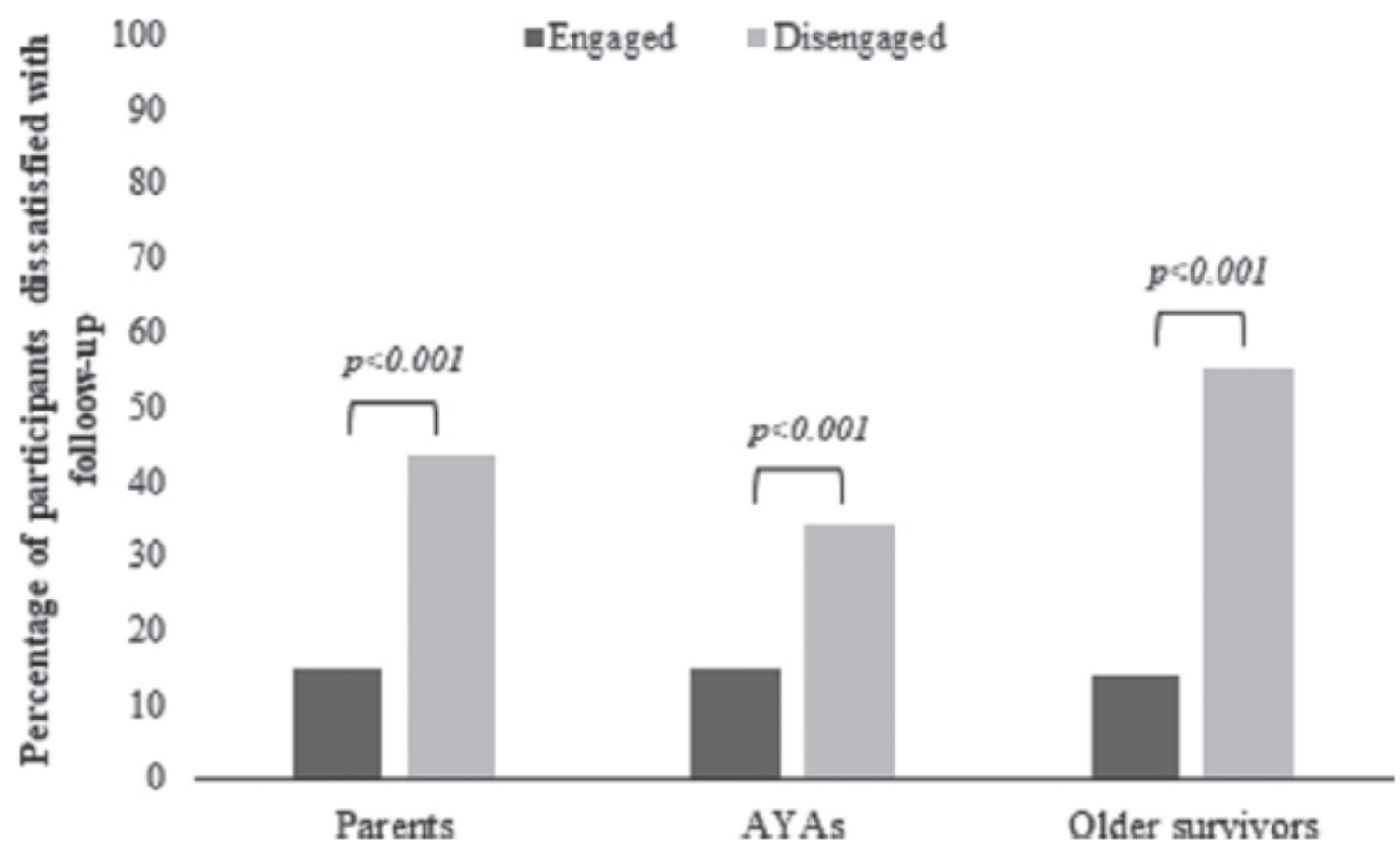

Figure 4. Participants' 'first choice' and willingness to see physicians for follow-up

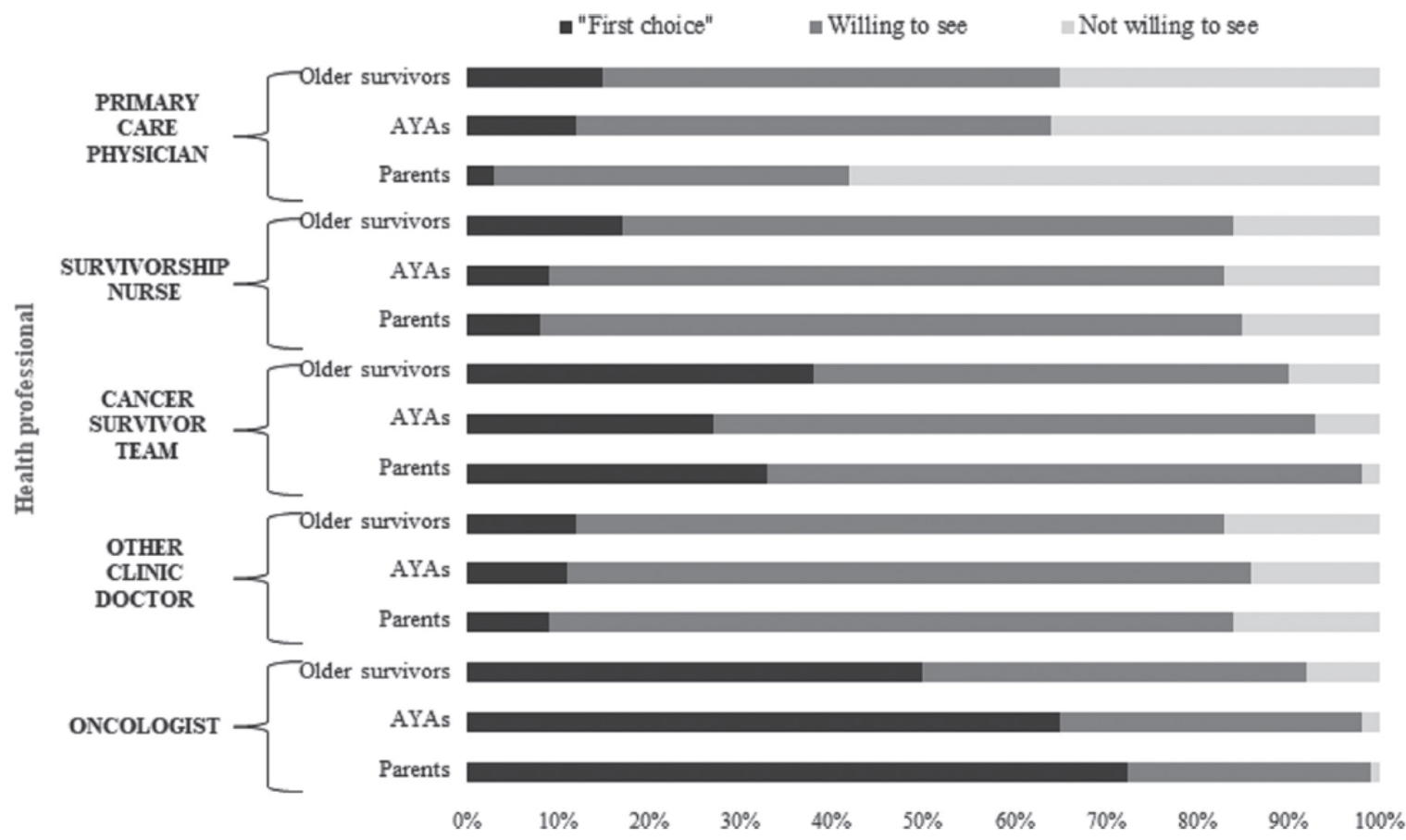

